\title{
Tuning towards dynamic freezing using a two-rate protocol
}

\author{
Satyaki Kar, Bhaskar Mukherjee, and K. Sengupta \\ Theoretical Physics Department, Indian Association for the Cultivation of Science, Jadavpur, Kolkata-700032, India.
} (Dated: September 5, 2021)

\begin{abstract}
We study periodically driven closed quantum systems where two parameters of the system Hamiltonian are driven with frequencies $\omega_{1}$ and $\omega_{2}=r \omega_{1}$. We show that such drives may be used to tune towards dynamics induced freezing where the wavefunction of the state of the system after a drive cycle at time $T=2 \pi / \omega_{1}$ has almost perfect overlap with the initial state. We locate regions in the $\left(\omega_{1}, r\right)$ plane where the freezing is near exact for a class of integrable and a specific non-integrable model. The integrable models that we study encompass Ising and XY models in $d=1$, Kitaev model in $d=2$, and Dirac fermions in graphene and atop a topological insulator surface whereas the non-integrable model studied involves the experimentally realized one-dimensional (1D) tilted Bose-Hubbard model in an optical lattice. In addition, we compute the relevant correlation functions of such driven systems and describe their characteristics in the region of $\left(\omega_{1}, r\right)$ plane where the freezing is near-exact. We supplement our numerical analysis with semi-analytic results for integrable driven systems within adiabatic-impulse approximation and discuss experiments which may test our theory.
\end{abstract}

PACS numbers: 73.43.Nq, 05.70.Jk, 64.60.Ht, 75.10.Jm

\section{INTRODUCTION}

The behavior of closed quantum systems driven out of equilibrium has attracted a lot of attention in recent times 1.3 . Such out-of-equilibrium systems explore a major region (if not all) of it's Hilbert space leading to a gamut of novel phenomena which have no equilibrium counterparts. For example, they exhibit universal scaling of excitation density when driven slowly through a quantum critical point ${ }^{4}$. Another such class of phenomenon involves dynamic transition where the free energy density of a quantum system after a quench develops non-analyticities leading to sharp cusp-like features in it's Lochsmidt echo pattern at specific times 10 -14; these transitions have no equilibrium analogs and can not be described by any local order parameter. Furthermore, the universality and the possibility of application of renormalization group (RG) methods to such driven systems has been a subject of recent research 15 -17; such analysis also leads to a several interesting features involving RG flow and universality of driven systems which are qualitatively different from their equilibrium counterparts.

A class of such dynamical phenomenon involve periodically driven quantum systems leading to multiple passages through their critical points during a drive cycle 18,22 . Such systems exhibit a wide class of dynamical phenomena which do not occur in their aperiodically driven counterparts. For example, they display signatures of Stuckelberg interference phenomenon in their excitations densities, dynamical correlation functions, and thermodynamic quantities such as work distribution $20[23$. Furthermore, periodically driven integrable systems show a separate class of dynamical transitions which originates from the change in topology of their Floquet spectrum and leaves its imprint on temporal behavior of local correlation functions 24 . Such driven systems also exhibit the phenomenon of dynamics induced freezing 21|22|25|26, this phenomenon manifests itself in a near unity overlap of the system wavefunction after single or multiple drive period(s) with the initial wavefunction at $t=0$. Such near-unity overlap signifying freezing occurs at specific discrete frequencies $\omega^{*}$ and is therefore qualitatively different from the trivial freezing that occurs for ultrafast drive frequencies.

In all of the phenomena mentioned above, the drive protocols used involve quench, ramp, or periodic change of a single parameter of the system Hamiltonian as a function of time. More recently, a separate class of protocols involving linear or power law ramp of two Hamiltonian parameters with distinct rate has been studied in details 22|2627. The novel features of dynamics induced by such two-rate ramp protocols include a generalization of the Kibble-Zurek scaling laws and possibility of reduction of excitation production as the system is ramped through a quantum critical point. The latter feature leads to better fidelity for quantum state preparation and provide a controlled way of reduction of heating during dynamics in critical systems 22 . To the best of our knowledge, the study of dynamics of closed quantum critical systems for such two rate protocols has been carried out for ramp protocols only; no such studies have been undertaken for periodic drives.

In this work, we aim to fill up this gap in the literature by studying the effect of two-rate periodic drive protocols on both integrable and non-integrable closed quantum systems. The drive protocol chosen involves periodic variation of two parameters of the system Hamiltonian with frequencies $\omega_{1}$ and $\omega_{2}=r \omega_{1}$. For integrable models, we choose a class of models represented by free fermionic Hamiltonian $H=\sum_{\vec{k}} \psi_{\vec{k}}^{\dagger} H_{\vec{k}} \psi_{\vec{k}}$, where $\psi_{\vec{k}}=\left(c_{\vec{k}}, c_{-\vec{k}}^{\dagger}\right)^{T}$ is the two component fermion field, $c_{\vec{k}}$ denotes fermionic annihilation operator, and $H_{\vec{k}}$ is a $2 \times 2$ matrix Hamiltonian density given by

$$
H_{\vec{k}}=\left[\lambda_{1}\left(\omega_{1} t\right)-b_{\vec{k}}\right] \tau_{3}+\lambda_{2}\left(r \omega_{1} t\right) \Delta_{\vec{k}} \tau_{1}
$$

where $\tau_{3}$ and $\tau_{1}$ are Pauli matrices in particle-hole space. Such free fermionic Hamiltonians represents Ising and XY models in $d=1^{28}$ and Kitaev model in $d=2^{29}$ 31. In addition, it also describes Dirac-like quasiparticles in graphene ${ }^{32}$ and on the surface of a topological insulators 33 . In what follows we shall carry out numerical analysis of this model in the context of XY model in $d=1$; we note, however, that our results shall be valid for any other representations of $H_{\vec{k}}$. 
The XY model in a transverse field constitute a model for of half-integer spins on a one-dimensional (1D) chain whose Hamiltonian is given by

$$
H_{\mathrm{XY}}=\sum_{\langle i j\rangle, \alpha=x, y} J_{\alpha} S_{i}^{\alpha} S_{j}^{\alpha}-h \sum_{i} S_{i}^{z},
$$

where $J_{x, y}$ are nearest coupling between $x$ and the $y$ components of the spins, $\langle i j\rangle$ indicates that $j$ is one of the neighboring sites of $i$, and $h$ is the transverse field. A standard Jordan-Wigner transformation ${ }^{3}$ maps Eq. 2 to Eq. 1 in $d=1$ with the identification

$$
\begin{aligned}
b_{k} & =\left(J_{x}+J_{y}\right) \cos (k), \quad \lambda_{1}=-h \\
\Delta_{k} & =-i \sin (k), \quad \lambda_{2}=\left(J_{x}-J_{y}\right)
\end{aligned}
$$

For non-integrable system, we choose the recently experimentally realized ultracold boson in their Mott insulating phase in the presence of an artificial electric field created either by shifting the center of the confining harmonic trap or by applying the spatially varying Zeeman magnetic field to the spinor bosons 2634 . The Hamiltonian of such bosons can be written as

$$
H_{1}=-J \sum_{\langle i j\rangle}\left(b_{i}^{\dagger} b_{j}+\text { h.c }\right)+\sum_{i}\left[\frac{U}{2} n_{i}\left(n_{i}-1\right)-E n_{i} i\right]
$$

where $b_{i}$ denotes boson annihilation operator on site $i, U$ is the on-site interaction, $E$ is the magnitude of the effective electric field in units of energy 3 , $J$ is hopping amplitude of bosons between the nearest site, and $n_{i}=b_{i}^{\dagger} b_{i}$ is the boson number operator. It has been shown in Ref. 34 that the low-energy properties of $H_{1}$ is captured by a dipole model whose Hamiltonian is given by

$$
H_{d}=-J \sqrt{n_{0}\left(n_{0}+1\right)} \sum_{\ell}\left(f_{\ell}^{\dagger}+f_{\ell}\right)+(U-E) \sum_{\ell} n_{\ell},(5)
$$

where $f_{\ell}=b_{i}^{\dagger} b_{j} / \sqrt{n_{0}\left(n_{0}+1\right)}$ is the dipole annihilation operator living on the link $\ell$ between sites $i$ and $j$, and $n_{0}$ denotes the boson occupation number of the parent Mott phase. These dipoles constitute bound states of a boson and a hole on adjacent sites $i$ and $j$ of the lattice and $n_{\ell}=f_{\ell}^{\dagger} f_{\ell}$ is the dipole number operator residing on the link $\ell$ between these sites. The dipole operators satisfy two additional constraints; first, at most one dipole can occur on any link $\left(n_{\ell} \leq 1\right)$ and second, the maximal number of dipoles on two adjacent links is also unity $\left(n_{\ell} n_{\ell \pm 1}=0\right)$. The model has two ground states; for $U \gg E$, the state correspond to the dipole vacuum which is same as the parent Mott state of the bosons while for $U \ll E$, the state corresponds to maximal number of dipoles which is a density wave state for the bosons. It was shown in Ref. 34 that these two ground states are separated by a transition at $E_{c}=U+1.31 J \sqrt{n_{0}\left(n_{0}+1\right)}$ which belongs to the Ising universality class. These phases of the model and the signature of the Ising transition between them have been verified in a recent experiment 38 . The non-equilibrium dynamics of the model for single parameter quench, ramp and periodic protocols have also been studied ${ }^{26 / 36 / 37}$. In what follows, we shall vary the electric field $E$ and hopping amplitude $J$ of these models according to periodic protocols characterized by frequencies $\omega_{1}$ and $\omega_{2}=r \omega_{1}$ respectively.

The main results that we obtain from such a study are the following. First, we develop an analytical framework for studying dynamics for integrable models (Eq. 1) subjected to arbitrary two-rate periodic protocol characterized by frequencies $\omega_{1}$ and $\omega_{2}=r \omega_{1}$ using an adiabatic-impulse approximation 39 ; the formalism developed constitutes a generalization of the adiabatic-impulse approximation method to two-rate periodic protocols. We show that in the lowfrequency regime, where this approximation is expected to be accurate, our results match those obtained using exact numerics. Second, we chart out, both numerically and using adiabatic impulse approximation, the overlap between the wavefunctions at the end of a drive period $\left(T=2 \pi / \omega_{1}\right)$ and the initial ground state wavefunction as a function of $\omega_{1}$ and $r$. Our results demonstrate that the second drive frequency can be used as a tuning parameter to obtain dynamics induced freezing, i.e., a near-perfect overlap between these wavefunctions. In particular, for the integrable models studied here, we show that $r=1$ seems to be the best choice for obtaining dynamic freezing and provide a semi-analytic explanation of this result. Third, we compute equal-time correlation functions of the XY model as a function of $\omega_{1}$ and $r$ and discuss the signature of dynamics induced freezing in such correlation functions. Fourth, we study the dynamics of the tilted BoseHubbard model driven out of equilibrium via the two-rate protocol described above using exact diagonalization (ED) for finite size systems with $N \leq 16$. We show that such a drive leads to near-perfect dynamics induced freezing for several $\omega_{1}$ for $r=2$, provide a qualitative explanation of this phenomenon using a numerically supported effective two state model, and compute the dipole-dipole correlation functions which bear the signature of such freezing phenomenon. Finally, we chart our experiments which can be used to test our theory.

The plan of the rest of the paper is as follows. In Sec. II we analyze the integrable fermionic Hamiltonian (Eq. 11) using both adiabatic-impulse approximation and exact numerics. This is followed by Sec. III where we study the dynamics of the dipole model (Eq. 5). Finally we summarize our results, discuss their experimental implications, and conclude in Sec. IV.

\section{INTEGRABLE MODELS}

In this section we shall study the dynamics of the free fermion Hamiltonian given by Eq. 1 subjected to the two rate periodic protocol. We first analyze such dynamics using an adiabatic-impulse approximation in Sec. II A This is followed by discussion of results from exact numerics in Sec. IIB. carried out using appropriate parameters for 1D XY model (Eqs. 2and 3), and their comparison with those obtained from 


\begin{tabular}{llll}
$\lambda_{\text {min }}$ & $\lambda_{c}$ & \\
\cline { 2 - 3 } & I & $\mathrm{t}_{1}$ &
\end{tabular} max

FIG. 1: Schematic representation of the time-evolution of the system with a critical point being located at $\lambda=\lambda_{c}$, Regions I, II and III are the adiabatic regimes separated by impulse regions at $t=t_{1} \equiv t_{1 \vec{k}}$ and $t=t_{2} \equiv t_{2 \vec{k}}$ when the system traverse the critical point. See text for details.

adiabatic-impulse approximation developed in Sec. II A

\section{A. Adiabatic-Impulse Approximation}

The basic assumption behind the adiabatic-impulse approximation lies in dividing the dynamics of a system subjected to a drive into two distinct regimes 39 . The first is the adiabatic regime where the rate at which the system Hamiltonian changes is small compared to the instantaneous energy gap; here the dynamics merely lead to accumulation of a phase of the system wavefunction. The second constitute the impulse regime where the rate of change of the Hamiltonian parameter is comparable to or larger than the instantaneous energy gap; in this regime, excitations are produced since the system can no longer follow the instantaneous ground state. In the context of the Hamiltonian given by Eq. 1, the latter region occurs when the system reaches the critical point. This approximation therefore becomes accurate for low-frequency drives where the impulse regime occurs near the critical point of the Hamiltonian. In what follows, we shall proceed with the assumption that $H_{\vec{k}}$ (Eq. 1) has an isolated critical point which the system crosses during the evolution; this assumption fails when the $H_{\vec{k}}$ has a gapless region such as for the Kitaev model242931. In this case, the adiabatic-impulse approximation can not be used in its present form. In this section, we shall only discuss cases where $H_{\vec{k}}$, given by Eq. 1 . does not have such gapless regions.

To treat the dynamics of Eq. 1 subjected to a two-rate periodic protocol using the adiabatic-impulse approximation, we first sketch the adiabatic and the impulse regions in Fig. 1 . We denote the instantaneous ground state at a given momentum $\vec{k}$ to be the ground sate of the $H_{\vec{k}}(t)$ (Eq. 1 : $\left|\psi_{\vec{k}}\right\rangle_{1}=$ $\left(u_{\vec{k}}^{0}(t), v_{\vec{k}}^{0}(t)\right)^{T}$, where

$$
\begin{aligned}
u_{\vec{k}}^{0}(t) & =\frac{\lambda_{2}(t) \Delta_{\vec{k}}}{D_{\vec{k}}(t)}, \quad v_{\vec{k}}^{0}(t)=-\frac{\left(\lambda_{1}(t)-b_{\vec{k}}\right)+E_{\vec{k}}(t)}{D_{\vec{k}}(t)} \\
E_{\vec{k}}(t) & =\sqrt{\left(\lambda_{1}(t)-b_{\vec{k}}\right)^{2}+\lambda_{2}^{2}(t) \Delta_{\vec{k}}^{2}} \\
D_{\vec{k}}(t) & =\sqrt{\left(\lambda_{1}(t)-b_{\vec{k}}+E_{\vec{k}}(t)\right)^{2}+\lambda_{2}^{2}(t) \Delta_{\vec{k}}^{2}}
\end{aligned}
$$

The corresponding instantaneous excited state wavefunction for any given $\vec{k}$ is given by $\left|\psi_{\vec{k}}(t)\right\rangle_{2}=\left(-v_{\vec{k}}^{0}(t), u_{\vec{k}}^{0}(t)\right)^{T}$. Using Eq. 6, one can write down the wavefunction of the driven system at any time $t$ and for a given $\vec{k}$ as

$$
\left|\psi_{\vec{k}}(t)\right\rangle=b_{1 \vec{k}}^{\prime}(t)\left|\psi_{\vec{k}}(t)\right\rangle_{1}+b_{2 \vec{k}}^{\prime}(t)\left|\psi_{\vec{k}}(t)\right\rangle_{2}
$$

with $\left(b_{1 \vec{k}}^{\prime}(0), b_{2 \vec{k}}^{\prime}(0)\right)=(1,0)$. In what follows, we shall use the notation $u_{\vec{k}}(0)=u_{\vec{k}}^{0}(T) \equiv u_{\vec{k}}^{0}$ and $v_{\vec{k}}(0)=v_{\vec{k}}^{0}(T) \equiv v_{\vec{k}}^{0}$.

To obtain expressions for $b_{1,2 \vec{k}}^{\prime}(t)$, we first identify the times $t_{1 \vec{k}}$ and $t_{2 \vec{k}}$ (Fig. 1) at which the system comes to an avoided level crossing for a given momenta $\vec{k}$. To this end, we note that the instantaneous energy gap of the system is given by

$$
\delta E_{\vec{k}}=2 \sqrt{\left(\lambda_{1}\left(\omega_{1} t\right)-b_{\vec{k}}\right)^{2}+\lambda_{2}^{2}\left(r \omega_{1} t\right) \Delta_{\vec{k}}^{2}} .
$$

This gap reaches a minima at $t=t_{1,2} \vec{k}$ at an avoided level crossing for which $\partial \delta E_{\vec{k}} / \partial t=0$. This leads to

$$
\dot{\lambda}_{1}\left(\omega_{1} t_{1 \vec{k}}\right)\left[\lambda_{1}\left(\omega_{1} t_{1 \vec{k}}\right)-b_{\vec{k}}\right]+\dot{\lambda}_{2}\left(\omega_{2} t_{1 \vec{k}}\right) \lambda_{2}\left(\omega_{2} t_{1 \vec{k}}\right) \Delta_{\vec{k}}^{2}=0
$$

where $\dot{x}=\partial_{t} x$. For simple enough protocols, it is possible to solve this equation to obtain an analytic expression for $t_{1,2 \vec{k}}$. For example if $\lambda_{1}=\lambda_{2}=A \cos \left(\omega_{1} t\right)$, one obtains $\omega_{1} t_{1 \vec{k}}=$ $\arccos \left[A b_{\vec{k}} /\left(A^{2}+\Delta_{\vec{k}}^{2}\right)\right]$ and $\omega_{1} t_{2 \vec{k}}=2 \pi-\omega_{1} t_{1 \vec{k}}$. In other, more complicated cases, one needs to solve Eq.9numerically to obtain $t_{1,2 \vec{k}}$.

Let us now consider the evolution of the system in region I (Fig. 1) for $t<t_{1 \vec{k}}$. In this region, the system is in the adiabatic regime and the wavefunction merely gathers a kinetic phase during evolution. The evolution operator in this regime is thus given by

$$
U_{1}(t, 0)=e^{-i \Omega_{1 \vec{k}}(t) \tau_{3}}, \Omega_{1 \vec{k}}(t)=\frac{1}{2} \int_{0}^{t} \delta E_{\vec{k}}\left(t^{\prime}\right) d t^{\prime}(10)
$$

Similarly, in region II for $t_{1 \vec{k}}<t<t_{2 \vec{k}}$ and III, for $t_{2 \vec{k}}<t \leq$ $T$, the evolution operators are given by

$$
\begin{aligned}
& U_{2}\left(t, t_{1 \vec{k}}\right)=e^{-i \Omega_{2 \vec{k}}(t) \tau_{3}}, \Omega_{2 \vec{k}}(t)=\frac{1}{2} \int_{t_{1 \vec{k}}}^{t} \delta E_{\vec{k}}\left(t^{\prime}\right) d t^{\prime} \\
& U_{3}\left(t, t_{2 \vec{k}}\right)=e^{-i \Omega_{3 \vec{k}}(t) \tau_{3}}, \Omega_{3 \vec{k}}(t)=\frac{1}{2} \int_{t_{2 \vec{k}}}^{t} \delta E_{\vec{k}}\left(t^{\prime}\right) d t^{\prime}
\end{aligned}
$$

Around $t=t_{1,2 \vec{k}}$, the system enters the impulse regions and excitations are produced. The key aspect of the adiabaticimpulse approximation is to approximate the impulse region to be exactly at $t_{1,2, \vec{k}}$. Near these points, the effective Hamiltonian has the form

$$
\begin{aligned}
\mathcal{H}_{\vec{k}}^{a}(t)= & {\left[\Lambda_{1 \vec{k}}+\dot{\lambda}_{1}\left(\omega_{1} t_{a \vec{k}}\right) \delta t_{a \vec{k}}\right] \tau_{3} } \\
& +\left[\Lambda_{2 \vec{k}}+\dot{\lambda}_{2}\left(\omega_{2} t_{a \vec{k}}\right) \delta t_{a \vec{k}} \Delta_{\vec{k}}\right] \tau_{1} \\
\Lambda_{1 \vec{k}}= & \lambda_{1}\left(\omega_{1} t_{a \vec{k}}\right)-b_{\vec{k}} \\
\Lambda_{2 \vec{k}}= & \Delta_{\vec{k}} \lambda_{2}\left(\omega_{2} t_{a \vec{k}}\right), \quad \delta t_{a \vec{k}}=t-t_{a \vec{k}},
\end{aligned}
$$


where $a=1,2$ for the two impulse regions. We note that

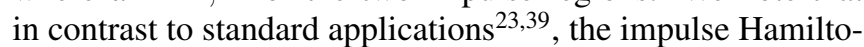
nian $\mathcal{H}_{\vec{k}}$ for the two-rate protocol is not of the Landau-Zener form; in contrast, it has time-dependence both in the diagonal and off-diagonal terms. To apply the Landau-Zener result, we therefore cast it in the standard form following the method suggested in Ref. 27. To this end, we first write $\mathcal{H}_{\vec{k}}(t)$ in terms of a new set of Pauli matrices as

$$
\mathcal{H}_{\vec{k}}^{a}(t)=\mu_{1 \vec{k}}^{a}\left(\delta t_{a \vec{k}}-t_{a \vec{k}}^{\prime}\right) \tilde{\tau}_{3}+\mu_{2 \vec{k}}^{a} \tilde{\tau}_{1}
$$

A comparison of Eqs. 12 and 13 shows that for both equations to be simultaneously correct, one needs

$$
\begin{aligned}
\tilde{\tau}_{3} \mu_{1 \vec{k}}^{a}= & \dot{\lambda}_{1}\left(\omega_{1} t_{a \vec{k}}\right) \tau_{3}+\dot{\lambda}_{2}\left(\omega_{2} t_{a \vec{k}}\right) \Delta_{\vec{k}} \tau_{1} \\
\tilde{\tau}_{1} \mu_{2 \vec{k}}^{a}= & \left(\Lambda_{1 \vec{k}}+\dot{\lambda}_{1}\left(\omega_{1} t_{a \vec{k}}\right) t_{a \vec{k}}^{\prime}\right) \tau_{3} \\
& +\left(\Lambda_{2 \vec{k}}+\dot{\lambda}_{2}\left(\omega_{2} t_{a \vec{k}}\right) t_{a \vec{k}}^{\prime} \Delta_{\vec{k}}\right) \tau_{1}
\end{aligned}
$$

The identities $\left(\operatorname{Det}\left[\tilde{\tau}_{1,3}\right]\right)^{2}=1$ and $\left\{\tilde{\tau}_{3}, \tilde{\tau}_{1}\right\}=0$, then leads to

$$
\begin{aligned}
\mu_{1 \vec{k}}^{a}= & \sqrt{\dot{\lambda}_{1}^{2}\left(\omega_{1} t_{a \vec{k}}\right)+\dot{\lambda}_{2}^{2}\left(\omega_{2} t_{a \vec{k}}\right) \Delta_{\vec{k}}^{2}} \\
\mu_{2 \vec{k}}^{a}= & {\left[\left(\Lambda_{1 \vec{k}}+\dot{\lambda}_{1}\left(\omega_{1} t_{a \vec{k}}\right) t_{a \vec{k}}^{\prime}\right)^{2}\right.} \\
& \left.+\left(\Lambda_{2 \vec{k}}+\dot{\lambda}_{2}\left(\omega_{2} t_{a \vec{k}}\right) t_{a \vec{k}}^{\prime} \Delta_{\vec{k}}\right)^{2}\right]^{1 / 2} \\
t_{a \vec{k}}^{\prime}= & -\left(\dot{\lambda}_{1}\left(\omega_{1} t_{a \vec{k}}\right) \Lambda_{1 \vec{k}}+\dot{\lambda}_{2}\left(\omega_{2} t_{a \vec{k}}\right) \Lambda_{2 \vec{k}}\right) / \mu_{1 \vec{k}}^{2} .
\end{aligned}
$$

For the Hamiltonian given by Eqs. 13 and 15 , the probability of excitation production can be read off as

$$
p_{\vec{k}}^{a}=\exp \left[-2 \pi \delta_{\vec{k}}^{a}\right], \quad \delta_{\vec{k}}^{a}=\left|\mu_{2 \vec{k}}^{a}\right|^{2} /\left(2\left|\mu_{1 \vec{k}}^{a}\right|\right)
$$

Using Eq. 16 , one can write down the unitary evolution matrices $\Sigma_{a}$ which propagates the wavefunction across $t=t_{a \bar{k}}$. The elements of $\Sigma_{a}$ can be expressed in terms of $p_{\vec{k}}^{a}$ and the Stokes phase $\tilde{\phi}_{s \vec{k}}^{a}$ as

$$
\begin{aligned}
\Sigma_{a} & =\left(\begin{array}{cc}
\sqrt{1-p_{\vec{k}}^{a}} e^{-i \tilde{\phi}_{s \vec{k}}^{a}} & -\sqrt{p_{\vec{k}}^{a}} \\
\sqrt{p_{\vec{k}}^{a}} & \sqrt{1-p_{\vec{k}}^{a}} e^{i \tilde{\phi}_{s \vec{k}}^{a}}
\end{array}\right), \\
\tilde{\phi}_{s \vec{k}}^{a} & =-\frac{\pi}{4}+\delta_{\vec{k}}^{a}\left(\ln \delta_{\vec{k}}^{a}-1\right)+\operatorname{Arg} \Gamma\left(1-i \delta_{\vec{k}}^{a}\right)
\end{aligned}
$$

where $\Gamma$ denotes the gamma function. Using Eqs. 10, 11, and 17. we finally obtain

$$
\begin{aligned}
\left(\begin{array}{c}
b_{1 \vec{k}}^{\prime} \\
b_{2 \vec{k}}^{\prime}
\end{array}\right)= & U_{3}\left(T, t_{2 \vec{k}}\right) \Sigma_{\vec{k}}^{2} U_{2}\left(t_{2 \vec{k}}, t_{1 \vec{k}}\right) \Sigma_{\vec{k}}^{1} U_{1}\left(t_{1 \vec{k}}, 0\right)\left(\begin{array}{c}
1 \\
0
\end{array}\right) \\
= & \left(\begin{array}{cc}
q_{+\vec{k}} & -q_{-\vec{k}}^{*} \\
q_{-\vec{k}} & q_{+\vec{k}}^{*}
\end{array}\right)\left(\begin{array}{c}
1 \\
0
\end{array}\right)=\left(\begin{array}{c}
q_{+\vec{k}} \\
q_{-\vec{k}}
\end{array}\right) \\
q_{+\vec{k}}= & e^{-i \Omega_{3 \vec{k}}}\left[\sqrt{\left(1-p_{\vec{k}}^{1}\right)\left(1-p_{\vec{k}}^{2}\right)} e^{-i \sum_{a=1}^{2}\left(\Omega_{a \vec{k}}+\tilde{\phi}_{s \vec{k}}^{a}\right)}\right. \\
& \left.-\sqrt{p_{\vec{k}}^{1} p_{\vec{k}}^{2}} e^{i\left(\Omega_{2 \vec{k}}-\Omega_{1 \vec{k}}\right)}\right]
\end{aligned}
$$

$$
\begin{aligned}
& q_{-\vec{k}}=e^{i \Omega_{3 \vec{k}}}\left[\sqrt{\left(1-p_{\vec{k}}^{2}\right) p_{\vec{k}}^{1}} e^{i\left(\tilde{\phi}_{s \vec{k}}^{2}+\Omega_{2 \vec{k}}-\Omega_{1 \vec{k}}\right)}\right. \\
& \left.+\sqrt{\left(1-p_{\vec{k}}^{1}\right) p_{\vec{k}}^{2}} e^{-i\left(\tilde{\phi}_{s \vec{k}}^{1}+\Omega_{1 \vec{k}}+\Omega_{2 \vec{k}}\right)}\right] \\
& \left|\psi_{\vec{k}}(T)\right\rangle=\left(\begin{array}{c}
q_{+\vec{k}} u_{\vec{k}}^{0}-q_{-\vec{k}} v_{\vec{k}}^{0} \\
q_{+\vec{k}} v_{\vec{k}}^{0}+q_{-\vec{k}} u_{\vec{k}}^{0}
\end{array}\right)=\left(\begin{array}{c}
u_{\vec{k}}(T) \\
v_{\vec{k}}(T)
\end{array}\right)
\end{aligned}
$$

Eq. 18 is the central result of this section. It allows us to express the final wavefunction at the end of a drive period for an arbitrary two-rate drive protocol. We note that the expressions of $q_{ \pm \vec{k}}$ obtained in Eq. 18 constitutes a generalization of its single-rate protocol counterpart 39 . Furthermore, it allows us to obtain semi-analytic expressions for excitation density and all fermionic correlators at the end of the drive. In the context of XY model in a transverse field where the transverse spin correlators can be expressed in terms of fermionic correlators $^{34}$, Eq. 18 allows us to obtain analytic expressions for the transverse magnetization and other relevant spin correlation functions. In what follows, we shall be interested in equal-time fermionic correlation functions

$$
\begin{aligned}
C_{\vec{i}-\vec{j}}= & L^{-d} \sum_{\vec{k}}\left\langle\psi_{\vec{k}}(T)\right|\left(c_{\vec{k}}^{\dagger} c_{\vec{k}}+\text { h.c. }\right)\left|\psi_{\vec{k}}(T)\right\rangle \\
& \times \cos [\vec{k} \cdot(\vec{i}-\vec{j})] \\
= & L^{-d} \sum_{\vec{k}}\left|v_{\vec{k}}(T)\right|^{2} \cos [\vec{k} \cdot(\vec{i}-\vec{j})] \\
F_{\vec{i}-\vec{j}}= & L^{-d} \sum_{\vec{k}}\left\langle\psi_{\vec{k}}(T)\right|\left(c_{k} c_{-k}+\text { h.c. }\right)\left|\psi_{\vec{k}}(T)\right\rangle \\
& \times \sin [\vec{k} \cdot(\vec{i}-\vec{j})] \\
= & L^{-d} \sum_{\vec{k}}\left(u_{\vec{k}}^{*}(T) v_{\vec{k}}(T)+\text { h.c. }\right) \sin [\vec{k} \cdot(\vec{i}-\vec{j})]
\end{aligned}
$$

and the excitation density $n_{d}$

$$
n_{d}=1-F, \quad F=L^{-d} \sum_{\vec{k}}\left|\left\langle\psi_{\vec{k}}^{0} \mid \psi_{\vec{k}}(T)\right\rangle\right|^{2}
$$

where $F$ is the wavefunction overlap at the end of the drive which approaches close to unity for dynamics induced freezing, the sum over momenta extends over half of the Brillouin zone, and $L$ is the linear dimension of the system. These quantities can be expressed in terms of $q_{ \pm \vec{k}}$ and the instantaneous eignstates at $t=T, u_{\vec{k}}^{0}$ and $v_{\vec{k}}^{0}$, using Eqs. 18 and 7 , as

$$
\begin{aligned}
n_{d}= & 1-F=L^{-d} \sum_{\vec{k}}\left|q_{-\vec{k}}\right|^{2} \\
C_{\vec{i}-\vec{j}}= & L^{-d} \sum_{\vec{k}}\left|q_{-\vec{k}} u_{\vec{k}}^{0}+q_{+\vec{k}} v_{\vec{k}}^{0}\right|^{2} \cos [\vec{k} \cdot(\vec{i}-\vec{j})] \\
F_{\vec{i}-\vec{j}}= & L^{-d} \sum_{\vec{k}}\left[\left(q_{+\vec{k}}^{*} u_{\vec{k}}^{0}-q_{-\vec{k}}^{*} v_{\vec{k}}^{0}\right)\left(q_{-\vec{k}} u_{\vec{k}}^{0}+q_{+\vec{k}} v_{\vec{k}}^{0}\right)\right. \\
& + \text { h.c. }] \sin [\vec{k} \cdot(\vec{i}-\vec{j})]
\end{aligned}
$$

In the next section, we shall use Eq.22 to discuss the behav- 


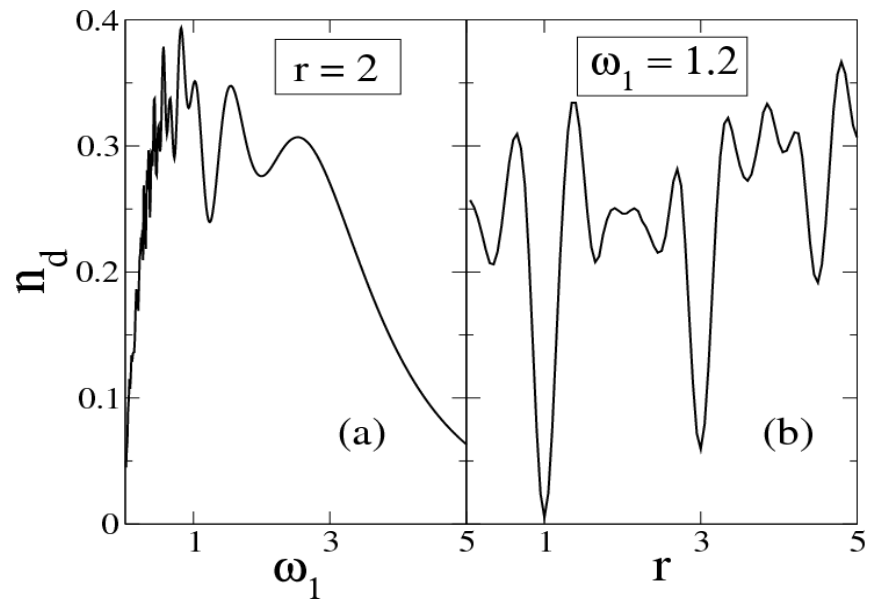

FIG. 2: (a) Plot of the defect density $n_{d}$ of the $1 \mathrm{D}$ XY model in a transverse field as a function of the drive frequency $\omega_{1}$ for $r=2$. Here we have chosen $J_{x}+J_{y}=1, J_{x}-J_{y}=\cos \left(r \omega_{1} t+\phi\right)$, and $h=A \cos \left(\omega_{1} t\right)$ with $A=4$ and $\phi=0$. (b) Plot of $n_{d}$ as a function of $r$ for $\omega_{1}=1.2$ and with all parameters same is (a). Note that $r=0$ correspond to the single parameter drive protocol. A clear signature of dynamic freezing is found at $r=1$ and $\omega_{1}=1.2$.

ior of magnetization, excitation density and correlation functions of the transverse field XY models as computed using adiabatic-impulse technique and compare with the exact numerics.

\section{B. Results for integrable models}

In this section, we discuss numerical results obtained by exact numerical solution of the driven Hamiltonian Eq. 1 and compare those obtained Sec. II A using generalized adiabaticimpulse approximation. To do this, we take advantage of the fact that $H_{k}$ represents a two-level Hamiltonian for each $(\vec{k},-\vec{k})$ pair. Thus for every given $\vec{k}$, it is possible to write a wavefunction $\left|\psi_{\vec{k}}(t)\right\rangle=\left(u_{\vec{k}}(t), v_{\vec{k}}(t)\right)^{T}$ which satisfies

$$
\begin{aligned}
i \partial_{t} u_{\vec{k}}(t) & =\left(\lambda_{1}(t)-b_{\vec{k}}\right) u_{\vec{k}}(t)+\lambda_{2}(t) \Delta_{\vec{k}} v_{\vec{k}}(t) \\
i \partial_{t} v_{\vec{k}}(t) & =-\left(\lambda_{1}(t)-b_{\vec{k}}\right) v_{\vec{k}}(t)+\lambda_{2}(t) \Delta_{\vec{k}} u_{\vec{k}}(t),
\end{aligned}
$$

where we first choose the parameters $\lambda_{1(2)}(t), b_{\vec{k}}$, and $\Delta_{\vec{k}}$ to be those for XY model in a transverse field (Eq. 3) with $\lambda_{1}=A \cos \left(\omega_{1} t\right), \lambda_{2}=\cos \left(r \omega_{1} t+\phi\right)$, and $J_{x(y)}=(1+$ $\left.(-) \lambda_{2}(t)\right) / 2$. Note that our choice of parameters is equivalent to scaling all energy parameters of the Hamiltonian with $J_{x}+$ $J_{y}$ and all time scales with $\hbar /\left(J_{x}+J_{y}\right)$. A numerical solution of Eq. 23 enables us to obtain $\left|\psi_{k}(t)\right\rangle$ which leads to the defect density $n_{d}$ and the correlation functions $C_{i j}$ and $F_{i j}$ using Eqs. 20 and 19 at the end of a single drive period $T=2 \pi / \omega_{1}$.

The results of the numerical procedure described above is shown in Figs. 2, 3. and 4. In Fig. 2(a), we plot $n_{d}$ as a function of $\omega_{1}$ for $r=2$ while in Fig. 2(b), we plot $n_{d}$ as a function of $r$ for $\omega_{1}=1.2$. These plots clearly demonstrate that $n_{d}$ approaches zero close to $r=1$ and $\omega_{1}=1.2$; at this point, $\left|\psi_{\vec{k}}(T)\right\rangle$ has almost perfect overlap with $\left|\psi_{k}(0)\right\rangle$ for

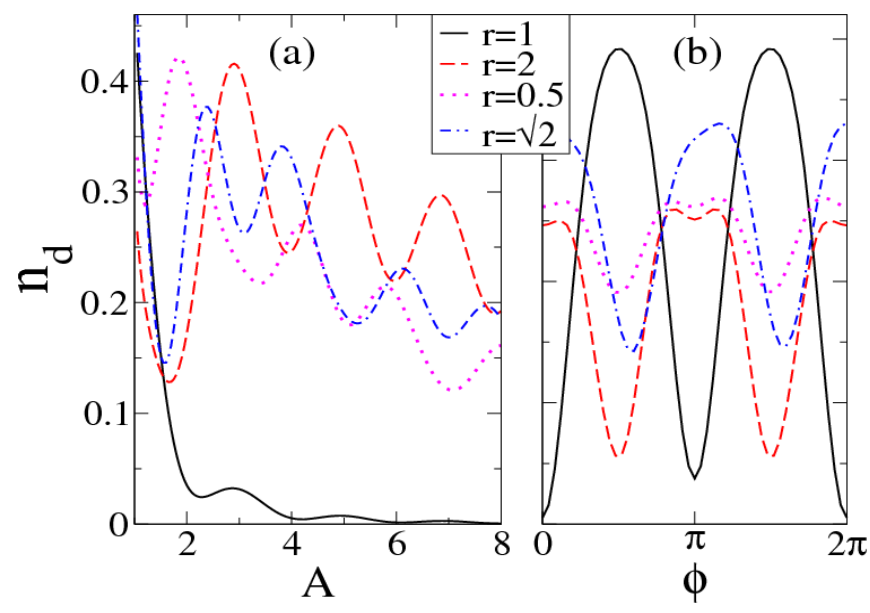

FIG. 3: (a) Variation of the defect density $n_{d}$ as a function of the drive amplitude for $\omega_{1}=1.2, \phi=0$, and several representative values of $r$. All other parameters are same in Fig. 2 (b) Variation of $n_{d}$ as a function of the initial phase $\phi$ of the drive for $A=4$.

all $k$ which signifies dynamic freezing. We note that such a freezing does not occur for a single parameter drive protocol (which corresponds to $r=0$ ) for any $\omega_{1}$; thus our results demonstrate that the second drive frequency, or equivalently $r$, for a two-parameter drive protocol can act as a tuning parameter to obtain such freezing. A plot of $n_{d}$ as a function of the drive amplitude $A$ (Fig 3 a) ) and the initial phase $\phi$ (Fig 3 (b)) for several representative values of $r$ indicates that $n_{d}$ indeed reaches a minimum of $r=1$ for all $A>1 ; n_{d}$ decreases rapidly with increasing $A$ for $r=1$ while it displays a slow oscillatory decay for other values of $r$. Further $n_{d}$ reaches a minimal value for $\phi=0$ as shown in Fig. 3(b). A comparison of these exact numerical results with those obtained using adiabatic-impulse approximation (Eq. 21) for $n_{d}$ is shown in Fig. 4. We find that the adiabatic-impulse approximation turns out to match the exact numerical results for $n_{d}$ for all $\omega_{1} \leq 1$ and for any $r$.

From Figs. 2, 3, and 4, it becomes apparent that the dynamics of $r=1$ is qualitatively different from that at $r \neq 1$ since it shows a rapid decay of $n$ with both $\omega_{1}$ and $A$. We now present a reason for this behavior. We first note that at $r=1$, the dynamics is controlled by $H_{\vec{k}}^{r=1}$ given by

$$
H_{\vec{k}}^{r=1}=\left(A f\left(\omega_{1} t\right)-b_{\vec{k}}\right) \tau_{3}+\Delta_{\vec{k}} f\left(\omega_{1} t\right) \tau_{1},
$$

where we have chosen $f\left(\omega_{1} t\right)=\cos \left(\omega_{1} t\right)$ for Figs. 2, 4. It turns out that $H_{\vec{k}}^{r=1}$ can be cast onto a form ${ }^{27}$

$$
H_{\vec{k}}^{r=1}=\alpha_{\vec{k}}\left(f\left(\omega_{1} t\right)-t_{1 \vec{k}}\right) \tilde{\tau}_{3}+\alpha_{2 \vec{k}} \tilde{\tau}_{1}
$$

where $\tilde{\tau}_{1,3}$ are new Pauli matrices which are related to $\tau_{1,3}$ by

$$
\begin{aligned}
& \alpha_{1 \vec{k}} \tilde{\tau}_{3}=A \tau_{3}+\Delta_{\vec{k}} \tau_{1} \\
& \alpha_{2 \vec{k}} \tilde{\tau}_{1}=\left(A t_{1 \vec{k}}-b_{\vec{k}}\right) \tau_{3}+\Delta_{\vec{k}} t_{1 \vec{k}} \tau_{1} .
\end{aligned}
$$

Using the properties that $\operatorname{Det} \tilde{\tau}_{1,3}=-1$ and $\left\{\tau_{3}, \tau_{1}\right\}_{+}=0$ 


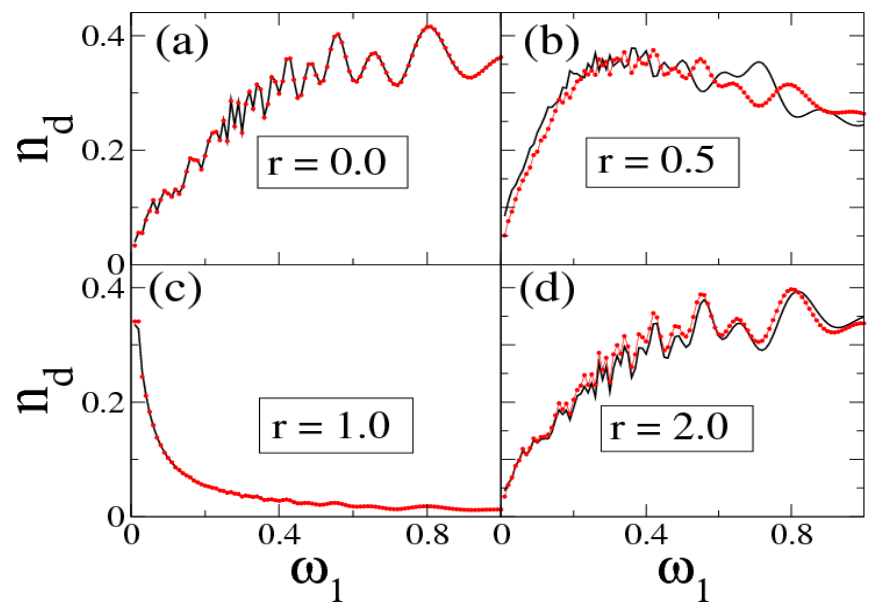

FIG. 4: Comparison of exact numerical result (black solid line) for variation of $n_{d}$ as a function of $\omega_{1}$ with that obtained from the adiabatic-impulse method (red dotted line) detailed in Sec. II A for (a) $r=0$, (b) $\mathrm{r}=0.5$, (c) $r=1$ and (d) $r=2$. All parameters are same as in Fig. 2

(where $\{. .\}_{+}$denotes anticommutator) one obtains

$$
\begin{aligned}
\alpha_{1 \vec{k}} & =-\sqrt{A^{2}+\Delta_{\vec{k}}^{2}} \\
t_{1 \vec{k}} & =A b_{\vec{k}} / \alpha_{1 \vec{k}}^{2}, \quad \alpha_{2 \vec{k}}=-\Delta_{\vec{k}} b_{\vec{k}} / \alpha_{1 \vec{k}}
\end{aligned}
$$

We note that such a transformation allows us to write $H_{\vec{k}}$ in terms of new Pauli matrices and with all time dependence being shifted to the diagonal term; such transformation exist for non-linear drive protocols only for $r=1$, i.e., when the off-diagonal and the diagonal terms of $H_{\vec{k}}^{r=1}$ are driven by identical drive protocol. The avoided level crossings occur at $t=t_{0 \vec{k}}$ and $t=T-t_{0 \vec{k}}$ where $f\left(\omega_{1} t_{0}\right)=b_{\vec{k}}$. Linearizing $H_{\vec{k}}^{r=1}$ around these point, we find that within adiabaticimpulse approximation, the Landau-Zener transition probability $p_{\vec{k}}$ can be read-off from Eq. 25 to be

$$
p_{\vec{k}}=e^{-\pi\left|\alpha_{2 \vec{k}}\right|^{2} /\left|\alpha_{1 \vec{k}} \dot{f}\left(\omega_{1} t_{0 \vec{k}}\right)\right|}
$$

We note that the maximal excitation production occurs from modes around $\vec{k}=\vec{k}_{0}$. Assuming that $\Delta_{\vec{k}} \sim\left|\vec{k}-\vec{k}_{0}\right|^{z}$ around $\vec{k}_{0}$ and $b_{\vec{k}_{0}} \neq 0$, we find that for $f_{1}\left(\omega_{1} t\right)=\cos \left(\omega_{1} t\right)$

$$
p_{\vec{k}} \simeq e^{-b_{\vec{k}}^{2}\left|\vec{k}-\vec{k}_{0}\right|^{2 z} /\left(\omega_{1} A^{3}\left|\sqrt{1-b_{\vec{k}}^{2} / A^{2}}\right|\right)}
$$

From Eq. 29 we find that $p_{k} \rightarrow 1$ as $A$ or $\omega_{1}$ increases; thus the excitation probability after one cycle of the drive $P_{\vec{k}} \sim p_{\vec{k}}\left(1-p_{\vec{k}}\right)$ rapidly decays to zero with increasing $A$ or $\omega_{1}$. It is instructive to compare this decay to the $r=0$ case where the off-diagonal term in time-independent. In this case one can read off $p_{\vec{k}}$ using the standard results provided in Ref. 39: $p_{\vec{k}}(r=0)=\exp \left[-\left|\vec{k}-\vec{k}_{0}\right|^{2 z} /\left(\omega_{1} A\left|\sqrt{1-b_{\vec{k}}^{2} / A^{2}}\right|\right)\right]$. Thus we find $p_{\vec{k}}$ approaches unity with increasing drive amplitude much more rapidly for $r=1$ which explains the almost

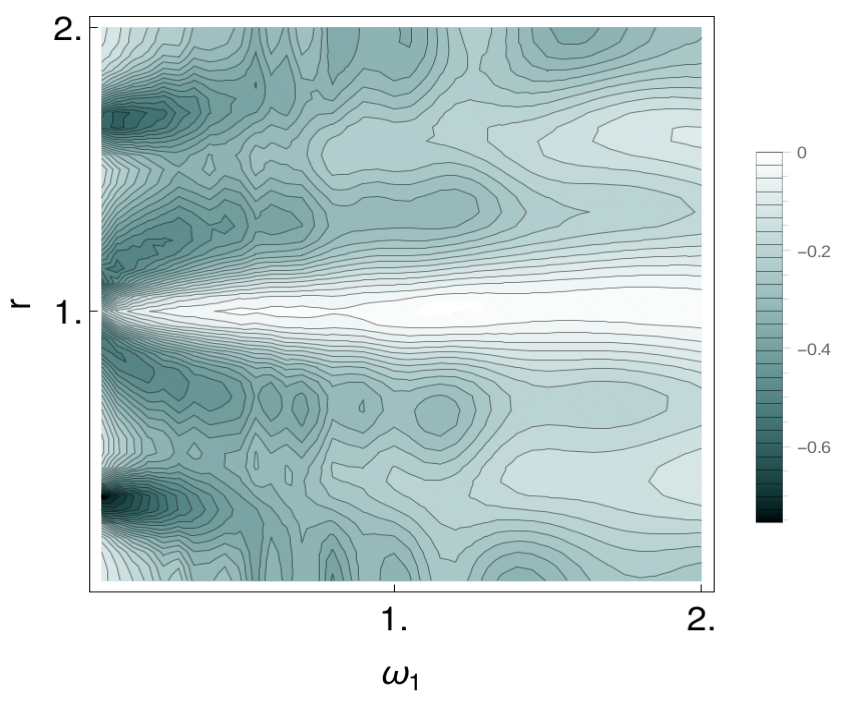

FIG. 5: Plot of the transverse magnetization $\delta m_{z}=m_{z}(T)-m_{z}(0)$ as a function of $r$ and $\omega_{1}$. The dark patch at $r=1$ is a signature of the dynamical freezing phenomenon which leads to $\delta m_{z}=0$.

monotonic decay of $P_{\vec{k}}$ and hence $n_{d}$ for $r=1$.

Finally we plot the change in transverse magnetization at $t=T, \delta m_{z}(T)=m_{z}(T)-m_{z}(0)$ for the XY model as a function of $r$ and $\omega_{1}$ in Fig. 5. We note that this magnetization can be easily obtained from the expressions of the correlation function $C_{\vec{i}-\vec{j}}$ given in Eq. 19

$$
m_{z}(T)=2 L^{-d} \sum_{\vec{k}}\left|v_{\vec{k}}(T)\right|^{2}-1=2 C_{0}(T)-1 .
$$

We note $\delta m_{z}$ vanishes if dynamic freezing occurs; such a vanishing of $\delta m_{z}$ is clearly seen along the $r=1$ line in Fig.5

\section{TITLED BOSE-HUBBARD MODEL}

In this section, we shall study the two-rate periodic drive protocol for the tilted Bose-Hubbard model whose Hamiltonian is given by Eq. 4 We shall analyze the dynamics of this model for $\hbar \omega_{1}, \hbar \omega_{2} \ll U, E$; in this regime, the dipole Hamiltonian given by Eq. 5 may be used to describe the dynamics of the bosons. In what follows, we shall make the electric field $E$ and the hopping $J$ time dependent according to the protocol

$$
\begin{aligned}
E(t) & =U-E_{0} \cos \left(\omega_{1} t\right) \\
J(t) & =J_{0}\left(1+\cos \left(r \omega_{1} t\right)\right)
\end{aligned}
$$

with $J(0)=1$. The electric field $E$ may be made timedependent by varying the Zeeman field used to generate it as a function of time ${ }^{38}$ while the detailed method creating a time dependent $J$ in realistic experimental situation has been discussed in Ref. 40. Following the convention used in previous studies on this model, we shall choose $U / J(0)=40$, $E_{0} / J(0)=10$ and $n_{0}=1$ so that the dynamics takes the system through the critical point located $E_{c}=U+1.83{ }^{34}$. 


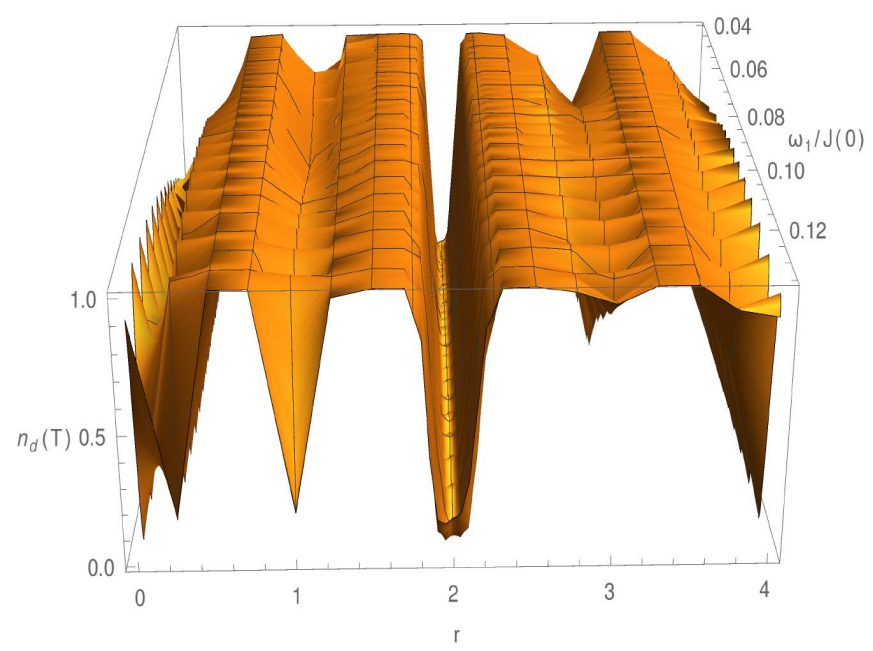

FIG. 6: Plot of the excitation density $n_{d}=1-F$ at $t=T$ as a function of $\omega_{1}$ and $r$ for $N=12, E(0)=30 J(0)$ and $U=40 J(0)$. Note that $n_{d} \simeq 0$ for $r=2$ independent of $\omega_{1}$.

To solve for the dynamics, we obtain the instantaneous eigenvalues and eigenvectors of the Hamiltonian $H_{d}$ given by Eq. 5 with $J(t)=J(0)$ and $E(t)=E(0)$ using exact diagonalization (ED) for a bosonic chain with $N \leq 16$ sites. We denote these many-body states by $|m\rangle$; they satisfy $H_{d}[J(0), E(0)]|m\rangle=\epsilon_{m}|m\rangle$ with $\epsilon_{1}$ and $|1\rangle$ being the ground state energy and wavefunction respectively. Having obtained $|m\rangle$, the wavefunction $|\psi(t)\rangle$ at any time which satisfies the Schrodinger equation $i \hbar \partial_{t}|\psi(t)\rangle=$ $H_{d}[J(t), E(t)]|\psi(t)\rangle$, can be expressed as

$$
|\psi(t)\rangle=\sum_{m} c_{m}(t)|m\rangle
$$

To obtain $c_{m}(t)$, we write the time-dependent Hamiltonian $H_{d}$ at any instant as

$$
\begin{aligned}
& H_{d}[J(t), E(t)]=H_{d}[J(0), E(0)]-J_{0} \cos \left(r \omega_{1} t\right) \\
& \times \sum_{\ell}\left(f_{\ell}+f_{\ell}^{\dagger}\right)+E_{0}\left(1-\cos \left(\omega_{1} t\right)\right) \sum_{\ell} n_{\ell} \\
= & H_{d}[J(0), E(0)]-J_{0} \cos \left(r \omega_{1} t\right) \Lambda_{1} \\
& +E_{0}\left(1-\cos \left(\omega_{1} t\right)\right) \Lambda_{2} .
\end{aligned}
$$

where the operators $\Lambda_{1,2}$ can be read off as $\Lambda_{1}=\sum_{\ell} n_{\ell}$ and $\Lambda_{2}=\sum_{\ell}\left(f_{\ell}+f_{\ell}^{\dagger}\right)$. Since $|m\rangle$ are eigenstates of $H_{d}[J(0), E(0)]$, this allows us to write

$$
\begin{aligned}
\left(i \hbar \partial_{t}-\epsilon_{m}\right) c_{m}= & \sum_{p} c_{p}\left[E_{0}\left(1-\cos \left(\omega_{1} t\right)\right)\left\langle m\left|\Lambda_{2}\right| p\right\rangle\right. \\
& \left.-J_{0} \cos \left(r \omega_{1} t\right)\left\langle m\left|\Lambda_{1}\right| p\right\rangle\right]
\end{aligned}
$$

These equations are solved numerically for a chain of length $N \leq 16$ sites to obtain $c_{m}(t)$ and hence the wavefunction $|\psi(t)\rangle$. In our numerics, we find that all results have similar

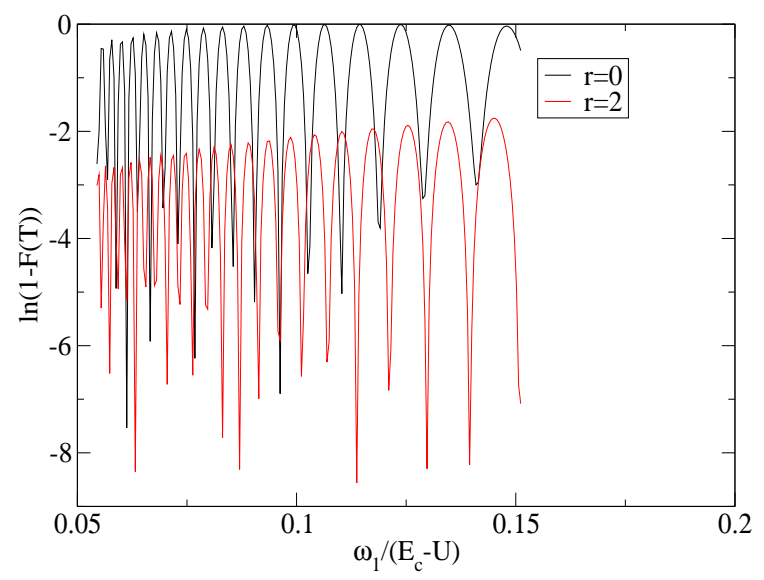

FIG. 7: Plot of $\ln (1-F)$ as a function of $\omega_{1}$ for $r=0$ and 2 for $N=14$. All other parameters are same as in Fig.6

features for $10 \leq N \leq 16$. Keeping in mind that experimental realization of such systems typically involves $N \leq 12$, we present our numerical results for $16 \leq N \leq 12$. In what follows, we shall use $|\psi(t=T)\rangle$ to compute wavefunction overlap $F$, the excitation density $n_{d}$, and the dipole correlation functions $C_{\ell \ell^{\prime}}$ which are given by

$$
\begin{aligned}
F & =|\langle\psi(T) \mid 0\rangle|^{2}=\left|c_{0}(T)\right|^{2}=1-n_{d} \\
C_{\ell \ell^{\prime}}^{d} & =\left\langle\psi(T)\left|n_{\ell} n_{\ell^{\prime}}\right| \psi(T)\right\rangle=\sum_{m p} c_{m}^{*}(T) c_{p}(T)\left\langle m\left|n_{\ell} n_{\ell^{\prime}}\right| p\right\rangle
\end{aligned}
$$

The results that we obtain from the above mentioned analysis is shown in Fig. 6 showing variation of $n_{d}(T)$ as a function of $\omega_{1}$ and $r$ for $N=12$. We find that for most $r, n_{d}$ is an oscillating function of $\omega_{1}$ which is qualitatively similar to its behavior for single rate drive protocols studied earliei ${ }^{26}$. However for $r=2, n_{d}$ displays a minimum for any $\omega_{1}$; for $r=2$, the value of $n_{d}$ always ranges between $10^{-2}$ and $10^{-9}$. This behavior can be clearly seen from Fig. 9 where $\ln (1-F)=\ln n_{d}$ is plotted as a function of $\omega_{1}$ with $N=14$ and for $r=0$ and $r=2$. The plot shows that $\ln n_{d} \leq-2$ for any $\omega_{1}$ when $r=2$; thus for $r=2$, the system displays dynamic freezing for a wide range of $\omega_{1}$. We find numerically that the overlap between the final and the initial wavefunctions is closer to unity for $r=2$ than for any other $r$ and $\omega_{1}$. We have checked that such dynamic freezing phenomenon is independent of the system size $N$ within the range of $N$ covered in our numerical analysis; $n_{d}$ shows a dip at $r=2$ for all $N \leq 16$.

To understand this phenomenon, we analyze the wavefunction overlap coefficients $\left|c_{n}(t)\right|^{2}$ as a function of time $t$ for both $r=0$ and $r=2$. A plot of these coefficients is shown in Fig. 8 for $r=0$ and $r=2$. The plot shows that for $r=0$, the system wavefunction $|\psi(t)\rangle$ has overlap with several eigenstates $|m\rangle$ (eigenstates of $H(0)$ ) as shown in left panel of Fig. 8. In particular, the change in overlap $|\psi(t)\rangle$ with $|m\rangle$ which 

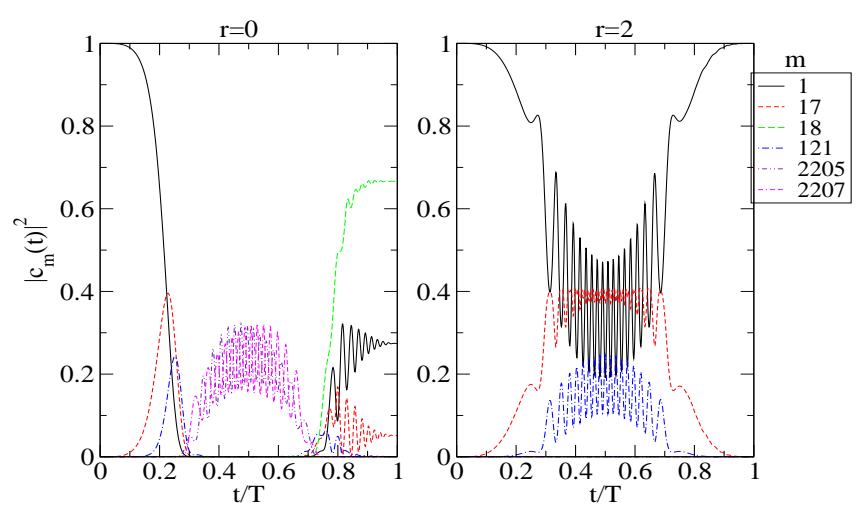

FIG. 8: Plot of the wavefunction overlap coefficients $\left|c_{m}(t)\right|^{2}$ as a function of $t$ with $\omega_{1} /\left(E_{c}-U\right)=0.107$ and $N=16$ for (a) $r=0$ and (b) $r=2$. We have only plotted coefficients for which $\left|c_{m}(t)\right|^{2} \geq 10^{-4}$. All other parameters are same as in Fig. 6

occur near $t \simeq T / 4,3 T / 4$ when the system traverses the critical point separating the dipole vacuum and maximal dipole ground states involves several $|m\rangle$ for $r=0$. Also around these points at $r=0, J$ is finite which facilitates transfer of weight of $|\psi(t)\rangle$ between different $|m\rangle$ (since these are states with different average dipole numbers $\left\langle m\left|\sum_{i} n_{i}\right| m\right\rangle$ ); consequently, one does not expect the system to return to its initial ground state at $t=T$ for generic $\omega_{1}$. In contrast, for $r=2$, we find that very few states $(m=1,121$, and 2207) contribute to $|\psi(t)\rangle$. Moreover at $t=T / 4,3 T / 4$, the transfer of weights occur only between states $|m=1\rangle$ (dipole vacuum ground state) and $|m=2207\rangle$ (maximal dipole state for $N=16$ ). Thus around these points, the dynamics is controlled by an effective two-level system similar to those analyzed in Sec. III In addition, we note $J(t)$ vanishes at the critical point for $r=2$ and remains very small around the critical point; thus these two levels undergo a nearly unavoided level crossing at $t=T / 4,3 T / 4$ since the matrix element of $H(t)$ between states with different dipole number is extremely small when $J(t) \simeq 0$. This feature, together with the fact only these two states are involved in dynamics of the system, ensures that $\left|c_{1}(t=T)\right|^{2} \simeq 1$ which leads to dynamic freezing. The degree of this overlap depends on $\omega_{1}$; however since $J(t)$ always vanishes at $t=T / 4,3 T / 4$, the overlap is always close to unity leading to $\ln (1-F) \leq-2$ for the entire range of $\omega_{1}$ studied in our numerics.

Finally, we plot the dipole correlation function between sites $\ell=2$ and $\ell^{\prime}=0, C_{20}^{d}(T) \equiv C_{2}^{d}(T)$, as a function of $r$ and $\omega_{1}$ in Fig. 9 . The choice of the site indices are motivated as follows. Due to the constraint $n_{\ell} \leq 1$ and $n_{\ell} n_{\ell+1}=0$ on the dipole Hamiltonian, $\left\langle n_{\ell}^{2}\right\rangle=\left\langle n_{\ell}\right\rangle$ and $\left\langle n_{\ell} n_{\ell+1}\right\rangle=0$ for any $\ell$. Thus the first non-trivial correlation between dipoles occur for $\left|\ell-\ell^{\prime}\right|=2$. We find that $C_{2}^{d}(T)$ is generically an oscillating function of $\omega_{1}$ for any $r$ expect at $r=2$ where its close to zero for all $\omega_{1}$. This behavior can be understood from the fact at $r=2,|\psi(t)\rangle$ is close to the starting ground state $|m=1\rangle$. Since we start our dynamics in a parameter regime

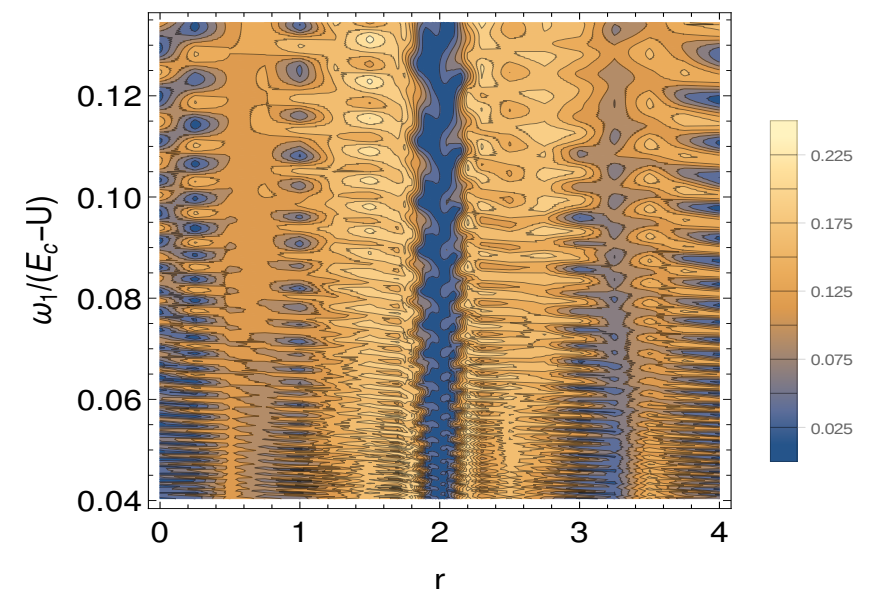

FIG. 9: Plot of the dipole correlation function $C_{2}^{d}(T)$ for $N=12$ as a function of $\omega_{1}$ and $r$ showing absence of correlation at $r=2$ for any $\omega_{1}$. All parameters are same as in Fig.6

where the ground state is dipole vacuum, $|m=1\rangle$ and hence $|\psi(t)\rangle$ has no dipole correlations.

\section{DISCUSSION}

In this work, we have studied periodic dynamics of a class of integrable [represented by free fermionic Hamiltonian (Eq. [1)] and an experimentally realizable non-integrable model [represented by bosonic Hamiltonian (Eq. [5]] using a two-rate protocol. For numerical purposes, we have chosen the 1D XY model in a transverse field as a representative example of the class of integrable model. For each of these models, we find that the second drive frequency $\omega_{2}=r \omega_{1}$ can act as tuning knob to achieve dynamical freezing.

For integrable models represented by Eq. 1. we obtain a semi-analytic expression for the wavefunction at the end of a drive cycle within the adiabatic-impulse approximation. Our results constitute a generalization of this method for application to integrable systems driven by arbitrary two-rate protocols. We compare the results obtained by this method, for low-enough drive frequencies where it is expected to be accurate, to exact numerics and find almost exact match between the two. Our numerics, for these integrable models, also reveal that the dynamics at $r=1$ is qualitatively different from those of $r \neq 1$; for $r=1$, dynamic freezing is obtained for a wide range of $\omega_{1}$. This behavior can be analytically explained at a qualitative level. We also show that the signature of such dynamic freezing can be easily deduced from fermionic correlators (or equivalently transverse magnetization of the XY model); the change in magnetization shows a near zero value at $r=1$ for a wide range of $\omega_{1}$.

For the tilted Bose-Hubbard model, we drive the applied electric field and the hopping parameter as a function of time with frequencies $\omega_{1}$ and $\omega_{2}=r \omega_{1}$ respectively. We find, similar to integrable models discussed earlier, that $r$ may act as a tuning parameter for obtaining dynamical freezing; 
for our protocol choice, we find that for $r=2$ the system undergoes dynamic freezing for a wide range of $\omega_{1}$. We show that for $r=2$, the wavefunction overlap $F$ satisfies $-2 \geq \ln (1-F) \geq-9$ which constitutes a much better overlap between the final and the initial wavefunction than what can be achieved in single-rate protocol ${ }^{26}$. We provide a semi-analytic justification of such near-perfect dynamic freezing and discuss its signature in dipole-dipole correlation function.

Experimental verification of our result could be most easily achieved by on-site parity of boson occupation $\left((-1)^{n_{i}}\right)$ measurement of bosons in the tilted Bose Hubbard model after a drive cycle. Such measurement have already been carried out for tilted bosons in equilibrium for $N=12$ sites 38 . Here we propose to start from the dipole vacuum $\left(n_{d}=0\right)$ state which correspond to odd $(n=1)$ boson occupation on all sites. We then propose to drive the system for one full period using the

1 A. Polkovnikov, K. Sengupta, A. Silva, and M. Vengalattore, Rev. Mod. Phys., 83, 863 (2011).

2 J. Dziarmaga, Adv. Phys. 59, 1063 (2010).

3 A. Dutta, U. Divakaran, D. Sen, B. K. Chakrabarti, T. F. Rosenbaum, and G. Aeppli, arXiv:1012.0653 (unpublished).

4 T. W. B. Kibble, J. Phys. A 9, 1387 (1976); W. H. Zurek, Nature (London) 317, 505 (1985).

5 A. Polkovnikov, Phys. Rev. B 72, 161201(R) (2005).

${ }^{6}$ K. Sengupta, D. Sen and S. Mondal, Phys. Rev. Lett. 100, 077204 (2008).

7 D. Sen, K. Sengupta and S. Mondal, Phys. Rev. Lett. 101, 016806 (2008).

8 A. Polkovnikov, Phys. Rev. Lett. 101, 220402 (2008).

9 A. Chandran, A. Erez, S. S. Gubser, and S. L. Sondhi, Phys. Rev. B 86, 064304 (2012).

${ }^{10}$ F. Pollmann, S. Mukerjee, A. M. Turner, and J. E. Moore Phys. Rev. Lett. 102255701 (2009).

11 M. Heyl, A. Polkovnikov, and S. Kehrein Phys. Rev. Lett. 110, 135704 (2013).

12 C. Karrasch and D. Schuricht, Phys. Rev. B, 87, 195104 (2013); N. Kriel, C. Karrasch, and S. Kehrein Phys. Rev. B 90, 125106 (2014)

${ }^{13}$ F. Andraschko, J. Sirker, Phys. Rev. B 89, 125120 (2014); E. Canovi, P. Werner, and M. Eckstein, Phys. Rev. Lett. 113, 265702 (2014); M. Heyl, Phys. Rev. Lett. 115, 140602 (2015).

${ }^{14}$ S. Sharma, U. Divakaran, A. Polkovnikov, and A. Dutta, arXiv:1601.01637 (unpublished).

15 L. M. Sieberer, S. D. Huber, E. Altman, and S. Diehl, Phys. Rev. Lett. 110, 195301 (2013).

16 S. de Sarkar, R. Sensarma, and K. Sengupta, Jour. Phys. Cond. Mat., 23, 305602 (2014).

17 A. Mitra and T. Giamarchi, Phys. Rev. Lett. 107150602 (2011); A. Mitra and T. Giamarchi, Phys. Rev. B 85075117 (2012).

${ }^{18}$ E. C. G. Stuckelberg, Helv. Phys. Acta 5, 369 (1932).

19 M. Demirplak and S. A. Rice, J. Chem. Phys. A 107, 9937 (2003); ibid, J. Chem. Phys. B 109, 6838 (2005).

${ }^{20}$ W. D. Oliver, Y. Yu, J. C. Lee, K. K. Berggren, L. S. Levitov, and T. P. Orlando, Science 310, 1653 (2005); M. S. Rudner, A. V. Shytov, L. S. Levitov, D. M. Berns, W. D. Oliver, S. O. Valenzuela, T. P. Orlando, Phys. Rev. Lett. 101, 190502 (2008)

21 A. Das, Phys. Rev. B, 82, 172402 (2010); S. Hegde, H. Katiyar, two-rate protocol given by Eq. 31 and measure the number of sites with even bosons. Since formation of a dipole in this case always lead to even $(n=0,2)$ boson occupation at adjacent sites, such a measurement provide us with information on dipole density and hence $1-F$. We predict that for $r=2$, almost no dipoles would be created for a wide range of $\omega_{1}$.

In conclusion, we have studied two-rate periodic protocols applied to a class of integrable models and an experimentally realizable non-integrable models. Our study reveals that the second drive frequency may be used as a tuning parameter to achieve dynamical freezing. We have provided a detailed analysis of this phenomenon, analyzed their signature in correlation functions, and suggested concrete experiments to test our theory.

Acknowledgement: KS thanks J.D. Sau for discussion on related projects. SK acknowledges financial support from CSIR, India, under Scientists' Pool Scheme No. 13(8764-A)/2015Pool.
T. S. Mahesh, and A. Das, Phys. Rev. B 90, 174407 (2014).

22 A. del Campo and K. Sengupta, Eur. Phys. Jour. Sp. Topics, 224, 189 (2015)

23 A. Dutta, A. Das, and K. Sengupta, Phys. Rev. E 92, 012104 (2015).

24 A. Sen, and K. Sengupta, arXiv:1511.03668 (unpublished).

${ }^{25}$ S. Mondal, D. Pekker and K. Sengupta EuroPhys. Lett. 100, 60007 (2012).

${ }^{26}$ U. Divakaran, and K. Sengupta, Phys. Rev. B 90, 184303 (2014).

27 J. D. Sau and K. Sengupta, Phys. Rev. B 90, 104306 (2014).

28 S. Sachdev, Quantum Phase Transitions (Cambridge University Press, Cambridge, England, 1999).

${ }^{29}$ A. Kitaev, Ann. Phys. (NY) 321, 2 (2006);

30 D. Sen, K. Sengupta, and S.Mondal, Phys.Rev. Lett 101, 016806 (2008).

31 X.-Y. Feng, G.-M. Zhang, and T. Xiang, Phys. Rev. Lett. 98, 087204 (2007).

32 A. H. Castro Neto, F. Guinea, N. M. R. Peres, K. S. Novoselov, and A. K. Geim, Rev. Mod. Phys. 81, 109 (2009); S. Das Sarma, S. Adam, E. H. Hwang, and E. Rossi, Rev. Mod. Phys. 83, 407 (2011); M. O. Goerbig, Rev. Mod. Phys. 83, 1193 (2011); D. N. Basov, M.M. Fogler, A. Lanzara, F.Wang, and Y. Zhang, Rev. Mod. Phys. 86, 959 (2014).

${ }^{33}$ M. Z. Hasan and C. L. Kane Rev. Mod. Phys. 82, 3045 (2010);X-L Qi and S-C Zhang, Rev. Mod. Phys. 83, 1057 (2011).

${ }^{34}$ S. Sachdev, K. Sengupta, and S. M. Girvin, Phys. Rev. B 66, 075128 (2002)

35 S. Pielawa, T. Kitagawa, E. Berg, and S. Sachdev, Phys. Rev. B 83, 205135 (2011); S. Pielawa, E. Berg, and S. Sachdev, Phys. Rev. B 86, 184435 (2012)

${ }^{36}$ K. Sengupta, S. Powell, and S. Sachdev, Phys. Rev.A 69, 053616 (2004).

37 M. Kolodrubetz, D. Pekker, B. K. Clark, and K. Sengupta, Phys. Rev. B 85, 100505 (2012).

38 J. Simon, W. Bakr, R. Ma, M. E. Tai, P. Preiss, and M. Greiner, Nature (London) 472, 307 (2011).

39 S. N. Shevchenko, S. Ashhab, and F. Nori, Phys. Rep. 492, 1 (2010).

40 S. de Sarkar, R. Sensarma, and K. Sengupta, Phys. Rev. B 92, 174529 (2015). 